Check for updates

Cite this: Chem. Sci., 2018, 9, 3523

Received 31st January 2018

Accepted 1st March 2018

DOI: $10.1039 / \mathrm{c} 8 \mathrm{sc} 00510 a$

rsc.li/chemical-science

\section{Porous aromatic framework with mesopores as a platform for a super-efficient heterogeneous Pd- based organometallic catalysis $\uparrow$}

\begin{abstract}
Li-Ping Jing, ${ }^{a}$ Jin-Shi Sun, ${ }^{a}$ Fuxing Sun, ${ }^{a}$ Peng Chen (D) *a and Guangshan Zhu (D) *ab
A strategy using a mesoporous amine-tagged porous aromatic framework (PAF70- $\left.\mathrm{NH}_{2}\right)$ to immobilize a palladium $(\mathrm{Pd})$-based molecular catalyst has been developed. The resulting immobilized catalyst PAF70-Pd, in which the framework is entirely constructed by phenyl rings linked with stable carboncarbon bonds, has high structural rigidity and stability. Compared with the known porous organic material immobilized Pd-based catalysts, PAF70-Pd has the highest Pd content so far. Moreover, PAF70Pd has extremely high catalytic activity with good size selectivity and very easy recyclability in catalyzing the Suzuki-Miyaura coupling reaction. In the current system, the catalyst loading could be as low as $0.001 \mathrm{~mol} \%$ and the TOF value could go up to $28800 \mathrm{~h}^{-1}$ which is far higher than those of the known porous organic material immobilized Pd-based catalysts. In order to elucidate the particularly high catalytic efficiency of PAF70-Pd, we prepared PAF1-Pd from PAF1- $\mathrm{NH}_{2}$ for comparison. PAF1-Pd has a higher Pd content than PAF70-Pd. However, due to the absence of large enough mesopores in PAF1$\mathrm{NH}_{2}$, PAF1-Pd has almost no catalytic activity under the same conditions, which definitely demonstrated that the intrinsic mesoporosity of PAF70- $\mathrm{NH}_{2}$ plays a crucial role in the superb catalytic efficiency of PAF70-Pd. This strategy to immobilize Pd-based molecular catalysts has very good expansibility to be applied in the immobilization of different organometallic catalysts into the pores of PAFs, which also has very high potential in the chemical and pharmaceutical industry.
\end{abstract}

\section{Introduction}

Organometallic catalysis, which uses a metal-based molecular catalyst to catalyze an organic reaction, is a very important field of organic chemistry. The excellent catalytic activity of the organometallic catalysts (metal-based molecular catalysts) has attracted intensive interest from chemists in diverse research fields. Thereinto, palladium (Pd)-based organometallic catalysts are versatile tools that can catalyze various organic reactions such as the Suzuki-Miyaura coupling reaction and Heck reaction. ${ }^{1-3}$ However, the high price of Pd-based catalysts and the Pd-residue in the products have greatly limited their applications in academia and industry. Immobilization of Pd-based catalysts onto some supported solid materials such as activated carbon, inert inorganic zeolites or organic polymers, typically by physical adsorption or chemical grafting (binding), is a good

${ }^{a}$ State Key Laboratory of Inorganic Synthesis and Preparative Chemistry, College of Chemistry, Jilin University, 2699 Qianjin Street, Changchun 130012, China. E-mail: pengchen@jlu.edu.cn

${ }^{b}$ Key Laboratory of Polyoxometalate Science of the Ministry of Education, Faculty of Chemistry, Northeast Normal University, Changchun 130024, China. E-mail: zhugs100@nenu.edu.cn

$\uparrow$ Electronic supplementary information (ESI) available: Experimental details including synthesis and experimental methods. See DOI: 10.1039/c8sc00510a method to solve the above problems. ${ }^{4-9}$ Although the rapid development of immobilization of Pd-based catalysts has been achieved, this field still suffers from reduced catalytic activity caused by poor accessibility or low metal loadings. ${ }^{\mathbf{1 0 - 2 9}}$

Using porous materials such as metal-organic frameworks (MOFs), covalent organic frameworks (COFs) or porous organic polymers (POPs) as supported materials began to appear in the last few decades, which is a good idea because of their porosity and high surface area. ${ }^{6,7,30-36}$ However, the intrinsic instability of MOFs and COFs or the flexibility of the frameworks of POPs makes this related research field still face many difficulties. In 2009, a new type of porous organic material with robust regular frameworks constructed entirely from rigid aromatic building blocks linked by stable covalent bonds, named porous aromatic frameworks (PAFs), was developed by our group and achieved intensive interest from researchers in diverse fields due to their wide range of structures and potential applications in gas sorption, ${ }^{37-45}$ separation, ${ }^{\mathbf{4 6 , 4 7}}$ catalysis, ${ }^{48-54}$ etc. Owing to their robust structure together with high stability in most organic solvents, PAFs are extremely suitable platforms for the catalysis of organic reactions. It's worth noting that, due to the presence of the Pd center and organic ligand, Pd-based organometallic catalysts usually have relatively large sizes. Hence, immobilization of Pd-based molecular catalysts into the porous materials often needs a large enough pore size. Most of the reported 
porous organic material immobilized Pd-based catalysts always suffer from low Pd utilization efficiency which might be due to that the pore space after introduction of the Pd-based catalyst is too small to accommodate the catalytic reaction. Apparently, for application of PAFs as the platforms for Pd-based organometallic catalysts, PAFs with large enough mesopores are needed. However, the synthesis of narrowly distributed mesoporous PAFs is still a challenge because of the interpenetration while using large-size monomers. Thus using PAFs as the platforms for covalent anchoring of organometallic catalysts into the pores still remains rare up to now. In this paper, we will make an attempt in this area.

Considering the need for large enough pore space for accommodating Pd-based molecular catalysts and the subsequent catalysis, in this paper, PAF70-NH with narrowly distributed mesopores which was recently reported by our group, ${ }^{55}$ was selected as the platform for Pd-based organometallic catalysis. In this paper, a strategy involving two post-synthesis modification steps for the introduction of the Pdbased organometallic catalyst into the pores of PAF70- $\mathbf{N H}_{2}$ was used for the synthesis of our desired material, and the catalytic performance of the desired material (PAF70-Pd) was systematically studied. In order to further demonstrate the importance of the mesopores in PAF70- $\mathbf{N H}_{2}$, another amine-tagged PAF (PAF1-NH $\mathbf{N H}_{2}$ without mesopores was also used as a platform to immobilize the same Pd-based molecular catalyst, affording PAF1-Pd for comparison with PAF70-Pd.

\section{Results and discussion}

\subsection{Synthesis of the materials}

Firstly, as shown in Scheme 1, via the pre-modification procedure used in our previous literature report, ${ }^{55}$ we synthesized PAF70-NH $\mathbf{N}_{2}$, which contains mesopores with $3.8 \mathrm{~nm}$ diameter

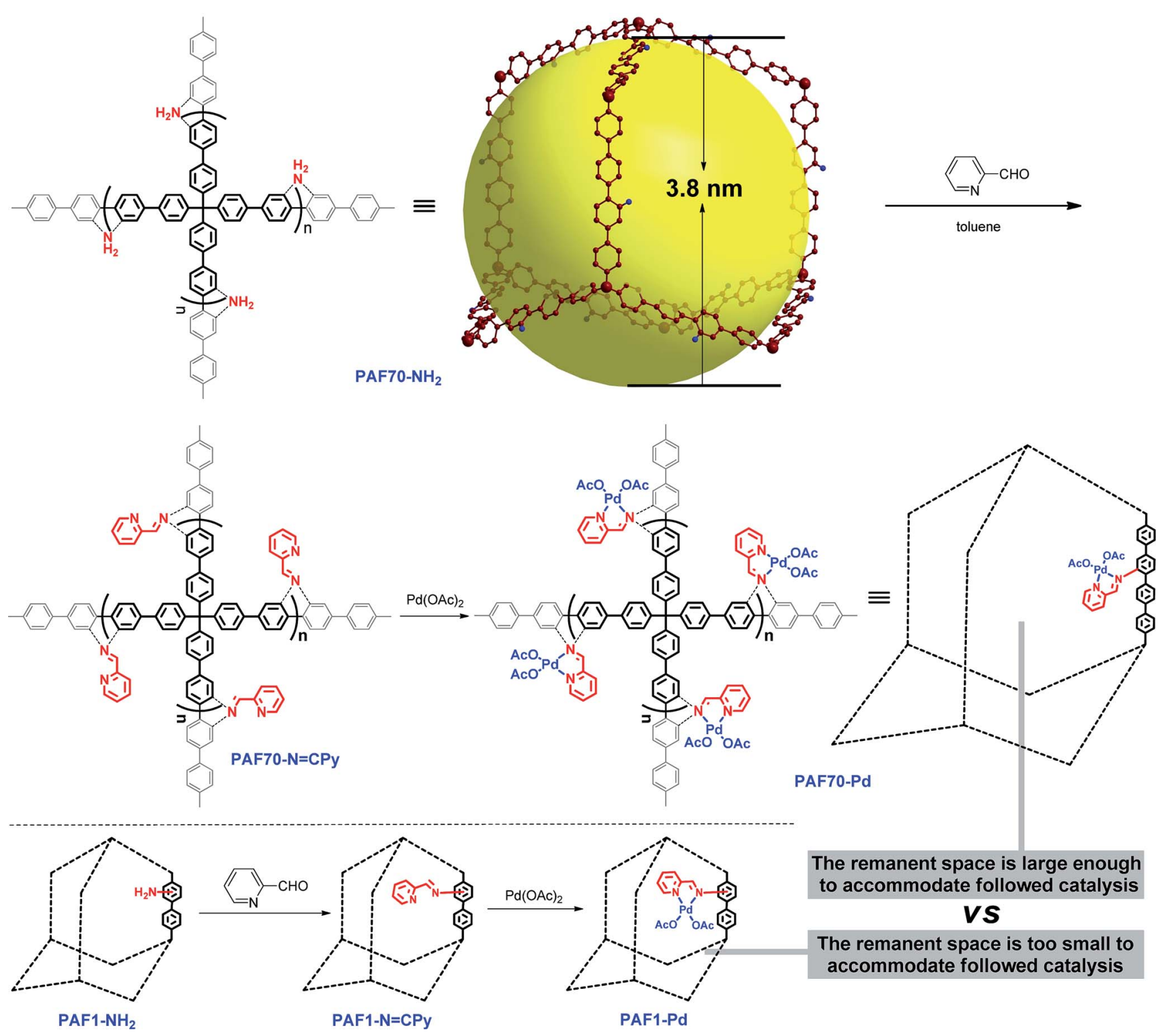

Scheme 1 Synthetic route to PAF70-Pd and PAF1-Pd. 
and amine anchors in the pores. Then, via the amine anchors using a condensation reaction with picolinaldehyde, the chelating ligand unit for Pd was introduced into the material, yielding the PAF which was named PAF70-N=CPy. After a second post-treatment of PAF70-N $=\mathbf{C P y}$ with palladium acetate, the PAF material containing the Pd-based molecular catalyst was obtained, which was named PAF70-Pd. It's worth noting that the $N, N$-bidentate ligand is one of the most versatile coordination systems in organometallic catalysis, which can coordinate with various metal ions and has been widely used in homogeneous catalysis. In addition, the post-synthesis modification was very facile and efficient. The above features of our synthetic method could expand the application value of the PAF material. In addition, for the purpose of comparison, we prepared PAF1- $\mathbf{N H}_{2}$ according to the literature report, ${ }^{51}$ and using a similar two-step post-synthesis method PAF1-Pd (the counterpart of PAF70-Pd) was prepared (Scheme 1).

\subsection{Characterization of the materials}

As shown in Fig. 1a, compared with the (Fourier transform infrared) FT-IR spectrum of PAF70- $\mathbf{N H}_{2}$, the strong attenuation of the characteristic double peaks of $-\mathrm{NH}_{2}$ (3464 and 3377 $\mathrm{cm}^{-1}$ ) and the appearance of the characteristic peaks of the Schiff base (the new peaks at around $1600 \mathrm{~cm}^{-1}$ ) in the FT-IR spectrum of PAF70-N $=\mathbf{C P y}$ indicated the formation of an imine bond and thus the successful construction of PAF70-N=CPy. In comparison with PAF70-N= CPy, in the FT-IR spectrum (Fig. 1a) of PAF70-Pd, the appearance and enhancement of the bands in the $1520-1750 \mathrm{~cm}^{-1}$ region could be attributed to the $\mathrm{C}=\mathrm{O}$
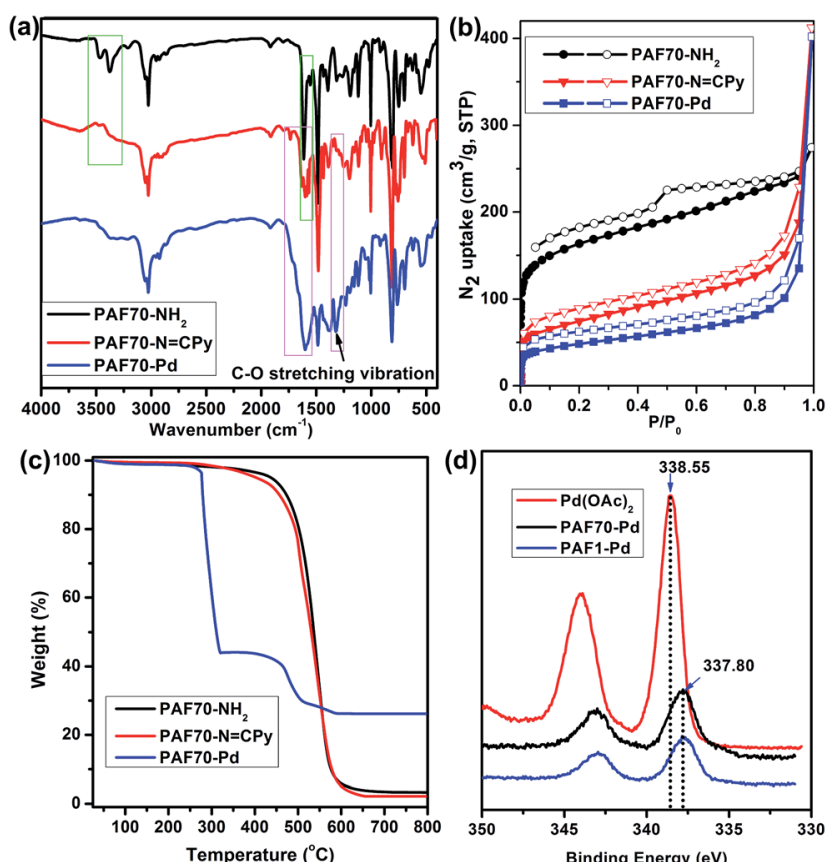

Fig. 1 FT-IR spectra (a), nitrogen adsorption (solid symbols)desorption (open symbols) isotherms measured at $77 \mathrm{~K}(\mathrm{~b})$, and TGA plots (c) of PAF70-NH $\mathrm{N}_{2}$ PAF70-N=CPy and PAF70-Pd. XPS spectra (d) of free $\mathrm{Pd}(\mathrm{OAC})_{2}, \mathrm{PAF} 70-\mathrm{Pd}$ and PAF1-Pd. stretching vibration and the new peaks at $1321 \mathrm{~cm}^{-1}$ could be attributed to the $\mathrm{C}-\mathrm{O}$ stretching vibration, which obviously indicated that PAF70-Pd was successfully obtained through our strategy. In addition, the successful synthesis of PAF1-Pd was also confirmed through a similar analysis of the FT-IR spectra (more details could be found in Fig. S15 in the ESI $\dagger$ ).

Nitrogen adsorption-desorption isotherms for the obtained materials were measured at $77 \mathrm{~K}$. As shown in Fig. 1b, at low relative pressures, PAF70-NH $\mathbf{N H}_{\mathbf{2}}, \mathbf{P A F 7 0 - N}=\mathbf{C P y}$ and PAF70-Pd all showed sharp uptakes, indicating the existence of micropores in the materials. It's worth noting that, in the desorption branch of PAF70- $\mathbf{N H}_{2}$, a relatively sharp hysteresis demonstrated the presence of narrowly distributed mesopores. Compared with PAF70-NH $\mathbf{N}_{2}$, the corresponding hysteresis disappeared in the desorption branches of PAF70-N=CPy and PAF70-Pd, which indicated the disappearance of the mesopores after postmodification of PAF70-NH $\mathbf{N H}_{2}$. The apparent surface area calculated from the Brunauer-Emmett-Teller (BET) model was 599 $\mathrm{m}^{2} \mathrm{~g}^{-1}$ for PAF70-NH $2,263 \mathrm{~m}^{2} \mathrm{~g}^{-1}$ for PAF70-N=CPy, and 172 $\mathrm{m}^{2} \mathrm{~g}^{-1}$ for PAF70-Pd. Through the change of pore size distributions calculated by non-local density functional theory (NLDFT), it was clear that the mesopores with a pore width of $3.8 \mathrm{~nm}$ of PAF70- $\mathbf{N H}_{2}$ disappeared in PAF70-Pd (see Fig. S10 in the ESI $\dagger$ ). The decrease of the BET surface area and the disappearance of mesopores from PAF70- $\mathbf{N H}_{2}$ to PAF70-Pd further proved the successful introduction of the Pd-based functional groups into the pores of the PAF.

Thermogravimetric analysis (TGA) was performed to test the thermal stabilities of the above PAF materials. As shown in Fig. 1c, PAF70-NH $\mathbf{N H}_{2}$ (black curve) and PAF70-N=CPy (red curve) showed similar TGA curves. There is almost no weight loss before $300^{\circ} \mathrm{C}$, which suggested the high thermal stability of PAF70-NH $\mathbf{N}_{2}$ and PAF70-N $=\mathbf{C P y}$. At about $400^{\circ} \mathrm{C}$, the framework decomposition started and when the temperature was above $500^{\circ} \mathrm{C}$ the decomposition became obvious. The $3.96 \mathrm{wt} \%$ residue for PAF70- $\mathbf{N H}_{2}$ and $2.15 \mathrm{wt} \%$ residue for $\mathbf{P A F 7 0 - N}=\mathbf{C P y}$ at $800^{\circ} \mathrm{C}$ could be ascribed to some palladium oxide residue which originated from the $\mathrm{Pd}$ catalysts in the preparation process of PAF70-NH2 $\mathbf{N}_{2}$ As shown in Fig. 1c, PAF70-Pd (blue curve) had a $56 \%$ weight loss at $277-320^{\circ} \mathrm{C}$. This weight loss could be attributed to the decomposition of both $N, N$-bidentate ligand and $\mathrm{AcO}^{-}$species which were directly connected to the Pd center. Compared with PAF70-N=CPy, PAF70-Pd showed lower stability, which might be due to that the Pd species could catalyze the cleavage of carbon-carbon bonds around the Pd centers in the PAF material. ${ }^{56}$ After a further obvious decomposition of the framework that started at $450^{\circ} \mathrm{C}$, there was a $26.2 \mathrm{wt} \%$ palladium oxide residue left at $800^{\circ} \mathrm{C}$. In addition, all the three PAF materials could not be dissolved or decomposed in almost all common solvents such as water, ethanol, dichloromethane, toluene, tetrahydrofuran, ethyl acetate, hexane, diethyl ether, etc. The high thermal stability and chemical stability made PAF70-Pd fully satisfy the demands of catalysis. The TGA analysis of PAF1-Pd can be found in the ESI (Fig. S17†). The Pd content was further determined by inductively coupled plasma (ICP) analysis, which revealed that $23.0 \mathrm{wt} \%$ of Pd was incorporated into PAF70-Pd and $25.1 \mathrm{wt} \%$ of 

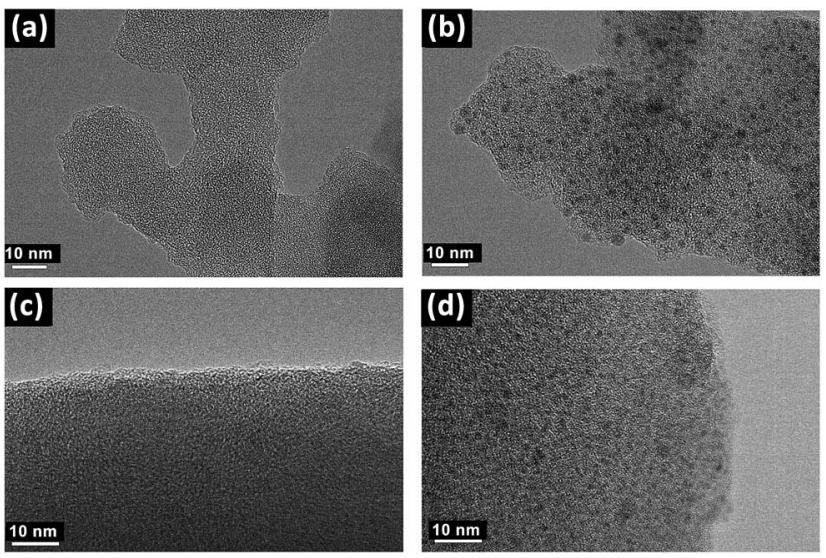

Fig. 2 TEM images of PAF70-N=CPy (a), PAF70-Pd (b), PAF1-N=CPy (c) and PAF1-Pd (d).

Pd was incorporated into PAF1-Pd. These were in agreement with the TGA analysis. Importantly, to the best of our knowledge, PAF70-Pd and PAF1-Pd have higher Pd contents than other reported porous organic material immobilized Pd catalysts, which significantly profits from that the pores of our PAF materials could endow high surface area for immobilizing the Pd coordination system.

In order to further investigate the incorporation of palladium within PAF70-N=CPy and PAF1-N $=\mathbf{C P y}$, X-ray photoelectron spectroscopy (XPS) was performed. As shown in Fig. 1d, the binding energy (BE) at $337.80 \mathrm{eV}$, assigned to the $\mathrm{Pd} 3 \mathrm{~d}_{5 / 2}$ orbital, indicated that the Pd species in PAF70-Pd and PAF1-Pd are present in the +2 state. Compared with the $\mathrm{BE}$ of $338.55 \mathrm{eV}$ for free $\mathrm{Pd}(\mathrm{OAc})_{2}$, the $\mathrm{BE}$ for $\mathrm{Pd}$ species in PAF70-Pd and
PAF1-Pd negatively shifted by $0.75 \mathrm{eV}$. This negative shift indicated that $\operatorname{Pd}(\mathrm{OAc})_{2}$ has strong coordination with the $\mathrm{N}, \mathrm{N}$ bidentate ligand in PAF70-N=CPy and PAF1-N $=\mathbf{C P y}{ }^{11,26}$

Transmission electron microscopys (TEM) images obviously showed the successful introduction of Pd species into the PAF materials. As shown in Fig. 2a and Fig. 2b, compared with PAF70-N=CPy, some evenly distributed black dots with a mean diameter of about $1 \mathrm{~nm}$ emerged in the TEM images of PAF70-Pd, indicating that the $\mathrm{Pd}$ species are uniformly dispersed in the frameworks of the PAF material, which was in accordance with the above analysis of the TGA curve of PAF70-Pd. Similarly, compared with PAF1-N=CPy (Fig. 2c), the TEM image of PAF1-Pd (Fig. 2d) also showed uniformly dispersed Pd species. This demonstrated that the Pd-based catalyst could also be anchored into the pores of the PAF1- $\mathbf{N H}_{2}$ material.

\subsection{Catalytic performance of PAF70-Pd}

After confirming the formation of the desired PAF70-Pd and PAF1-Pd, their catalytic properties were then studied. SuzukiMiyaura coupling reaction, one of the representative Pd-catalyzed reactions, was selected as the model reaction to study their catalytic performance. Firstly, some control experiments were conducted using $p$-bromonitrobenzene and phenylboronic acid as model substrates and PAF70-Pd as the catalyst. As shown in entries 1-3 of Table 1 , among the screened solvents $\left(\mathrm{CH}_{2} \mathrm{Cl}_{2}\right.$, $p$-xylene and $\mathrm{EtOH})$, EtOH gave the best results in terms of the reaction rate and yield of the current catalytic Suzuki-Miyaura coupling reaction. Increasing the reaction temperature from $25^{\circ} \mathrm{C}$ to $80^{\circ} \mathrm{C}$ improved the reaction rate significantly (Table 1 , entries 3-6). The catalyst loading screening showed that

Table 1 The control experiments for PAF70-Pd catalyzed Suzuki-Miyaura coupling reaction ${ }^{a}$

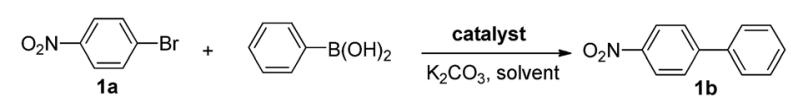

\begin{tabular}{|c|c|c|c|c|c|}
\hline Entry & Catalyst (catalyst loading) & Solvent & $T\left[{ }^{\circ} \mathrm{C}\right]$ & Time & Yield $^{b}$ \\
\hline 1 & PAF70-Pd (0.5 mol\%) & $\mathrm{CH}_{2} \mathrm{Cl}_{2}$ & 40 & $12 \mathrm{~h}$ & 0 \\
\hline 2 & PAF70-Pd (0.5 mol\%) & $p$-Xylene & 150 & $4 \mathrm{~h}$ & $92 \%$ \\
\hline 3 & PAF70-Pd (0.5 mol\%) & EtOH & 25 & $1 \mathrm{~h}$ & $92 \%$ \\
\hline 4 & PAF70-Pd (0.5 mol\%) & EtOH & 40 & $45 \mathrm{~min}$ & $96 \%$ \\
\hline 5 & PAF70-Pd (0.5 mol\%) & EtOH & 60 & $20 \mathrm{~min}$ & $95 \%$ \\
\hline 6 & PAF70-Pd (0.5 mol\%) & EtOH & 80 & $7 \mathrm{~min}$ & $97 \%$ \\
\hline $7^{c}$ & PAF70-Pd $(0.1 \mathrm{~mol} \%)$ & EtOH & 80 & $15 \mathrm{~min}$ & $96 \%$ \\
\hline $8^{c}$ & PAF70-Pd (0.01 mol\%) & EtOH & 80 & $25 \mathrm{~min}$ & $97 \%$ \\
\hline $9^{d}$ & PAF70-Pd (0.001 mol\%) & EtOH & 80 & $4 h$ & $93 \%$ \\
\hline 10 & No catalyst & EtOH & 80 & $12 \mathrm{~h}$ & 0 \\
\hline 11 & PAF70-N=CPy & EtOH & 80 & $12 \mathrm{~h}$ & 0 \\
\hline $12^{e}$ & The supernatant liquid of the EtOH suspension of PAF70-Pd and $\mathrm{K}_{2} \mathrm{CO}_{3}$ & EtOH & 80 & $12 \mathrm{~h}$ & 0 \\
\hline 13 & PAF1-Pd (0.01 mol\%) & EtOH & 80 & $25 \mathrm{~min}$ & $<5 \%$ \\
\hline
\end{tabular}

${ }^{a}$ Reaction conditions (unless otherwise noted): a solution of $1 \mathrm{a}(0.5 \mathrm{mmol})$, phenylboronic acid $(0.75 \mathrm{mmol}), \mathrm{K}_{2} \mathrm{CO}_{3}(1.0 \mathrm{mmol})$, and the catalysts (for entry 10, no catalyst was added; for entry 11, $1.2 \mathrm{mg}$ PAF70-N=CPy was added as the catalyst; for other entries, the catalysts were added at the indicated loadings based on Pd) in $2 \mathrm{~mL}$ of solvent was stirred at the indicated temperature for the indicated time. ${ }^{b}$ The isolated yield. ${ }^{c}$ The reaction scale was $2.5 \mathrm{mmol}$ of $1 \mathrm{1a} .{ }^{d}$ The reaction scale was $25.0 \mathrm{mmol}$ of $1 \mathrm{a} .{ }^{e} 1.2 \mathrm{mg}$ PAF70-Pd and $\mathrm{K}_{2} \mathrm{CO}_{3}(1.0 \mathrm{mmol})$ were immersed in $2 \mathrm{~mL}$ of $\mathrm{EtOH}$ for $2 \mathrm{~h}$ at $80^{\circ} \mathrm{C}$; after centrifugation, to the supernatant liquid were added $0.5 \mathrm{mmol} \mathrm{1a}, 0.75$ mmol phenylboronic acid and $1.0 \mathrm{mmol}$ $\mathrm{K}_{2} \mathrm{CO}_{3}$, then the resulting mixture was stirred at $80^{\circ} \mathrm{C}$ for $12 \mathrm{~h}$. 
increasing the catalyst loading could improve the reaction rate (Table 1, entries 6-9). It was exciting to note that when the catalyst loading of PAF70-Pd was reduced to $0.01 \mathrm{~mol} \%$, the current catalytic reaction could still occur rapidly (Table 1, entry 8) and when the catalyst loading of PAF70-Pd was reduced to $0.001 \mathrm{~mol} \%$, the current catalytic reaction could still occur smoothly (Table 1, entry 9). Under the best conditions (Table 1, entry 8) obtained from the above screenings, the current reaction could not occur without a catalyst (Table 1, entry 10). In addition, PAF70-N=CPy (Table 1 , entry 11 ) with the palladium residue in the material could not catalyze the reaction, indicating that the Pd residue from the preparation process in these materials has no catalytic activity. The above results demonstrated that PAF70-Pd is indeed an efficient catalyst for the Suzuki-Miyaura coupling reaction. Furthermore, the supernatant liquid of the EtOH suspension of PAF70-Pd showed no catalytic activity for the coupling reaction (Table 1 , entry 12) even in a much longer time, which indicated no leakage of catalytically active species from the PAF70-Pd catalyst during the catalysis process. Thus the current PAF70-Pd catalyzed reaction proceeds via a heterogeneous catalytic process.

For comparison, PAF1-Pd was then employed as the catalyst for the current Suzuki-Miyaura coupling reaction under the best conditions as shown in entry 8 of Table 1 . Compared with PAF70-Pd which gave a 97\% yield (Table 1, entry 8), PAF1-Pd showed almost no catalytic activity ( $<5 \%$ yield, Table 1 , entry 13$)$ under the same conditions which should be due to that the remnant space in the pores after introducing the Pd-catalyst was too small to accommodate the current catalysis. This comparison fully demonstrated the importance of the large enough mesopores in PAF70- $\mathbf{N H}_{2}$ for its application in immobilizing large-size metal-based molecular catalysts.

The catalytic performance of PAF70-Pd was further tested using a series of aryl bromides as the reaction substrates at a $0.01 \mathrm{~mol} \%$ catalyst loading. As shown in Table 2, bromobenzene 9 a (entry 9) or the substituted aryl bromides with either

Table 2 PAF70-Pd catalyzed Suzuki-Miyaura coupling reaction ${ }^{a}$

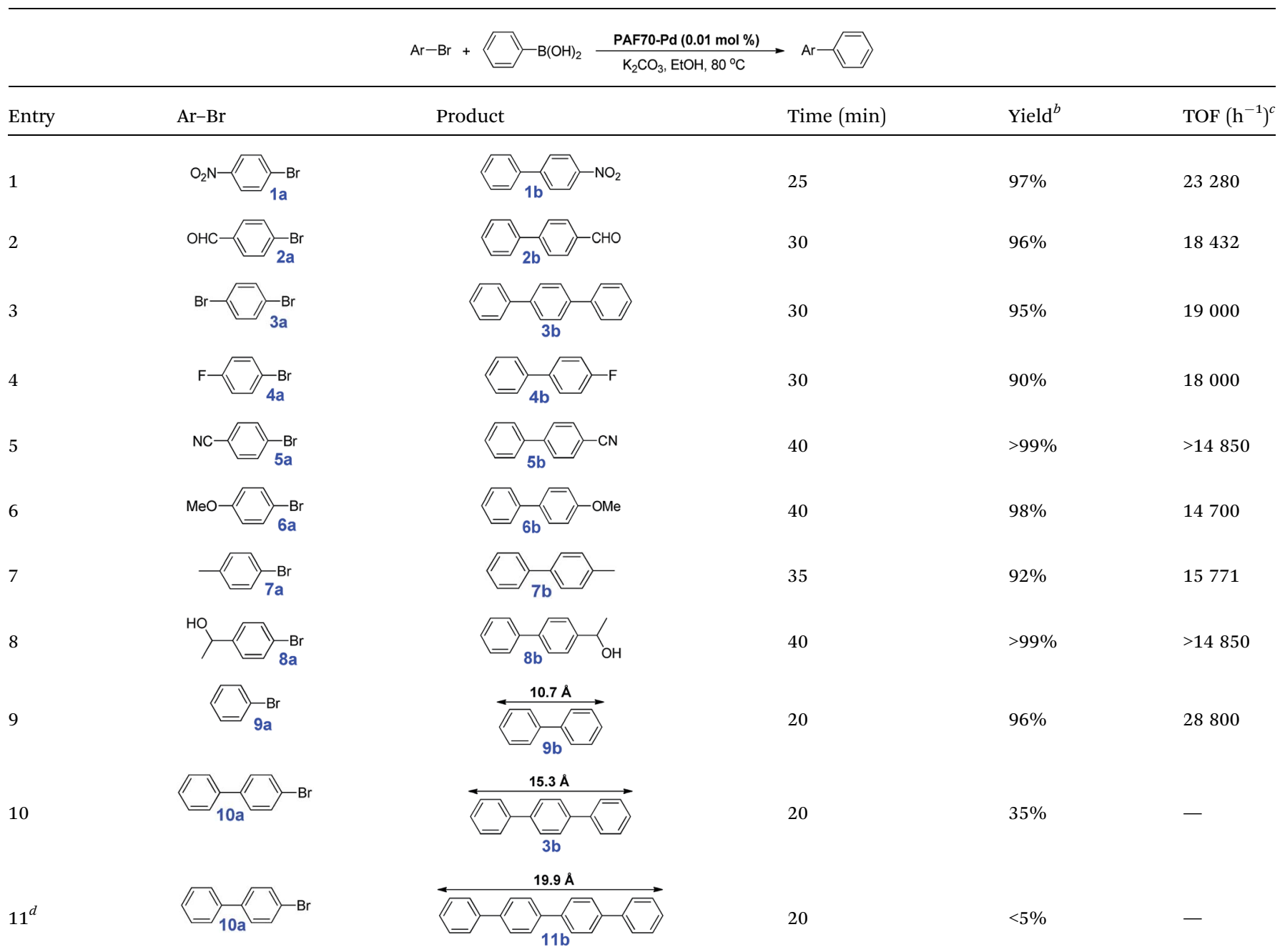

\footnotetext{
${ }^{a}$ Reaction conditions: a solution of $2.5 \mathrm{mmol} \mathrm{1a}, 3.75 \mathrm{mmol}$ phenylboronic acid, $5.0 \mathrm{mmol} \mathrm{K}_{2} \mathrm{CO}_{3}$ and PAF70-Pd $(0.01 \mathrm{~mol} \%)$ in $10 \mathrm{~mL}$ of EtOH was stirred at $80^{\circ} \mathrm{C}$ for the indicated time. ${ }^{b}$ The isolated yield. ${ }^{c}$ TOF $=$ (moles of product)/(moles of Pd in the catalyst $\times$ reaction time). ${ }^{d}$ For entry $11,4-$ biphenylboronic acid was used instead of phenylboronic acid.
} 
an electron-withdrawing group (such as $-\mathrm{NO}_{2}, \mathrm{CHO},-\mathrm{Br},-\mathrm{F}$ and - $\mathrm{CN}$, entries 1-5) or an electron-donating-group (such as-OMe, -Me and - $(\mathrm{OH}) \mathrm{CHCH}_{3}$, entries 6-8) afforded the cross-coupling products in excellent yields (up to $>99 \%$ ) with high turnover frequency (TOF) values (all $\geq 14700 \mathrm{~h}^{-1}$ ), demonstrating the wide generality and functional tolerance of the current system.

For the porous material immobilized catalysts with narrowly distributed pore size, the size selectivity is a signature feature of the catalyzed reaction which could occur in the pores. In order to investigate the size selectivity of PAF70-Pd, some contrast tests were performed as shown in entries 9-11 of Table 2 . Compared with 9a, which could smoothly transform to $\mathbf{9 b}$ completely (Table 2, entry 9), the larger-size 10a reacted more slowly under the same conditions in the same time (35\% yield, Table 2, entry 10). When 10a reacted with the larger-size 4-biphenylboronic acid, the reaction rate further decreased and even almost no product was obtained under the same conditions in the same time (Table 2, entry 11). The above size selectivity obviously indicated that the catalytic reaction could occur inside the pores of PAF70-Pd.

For heterogeneous catalysts, recyclability is an important factor. Hence the recyclability of PAF70-Pd as the catalyst was tested by subjecting it to 3 cycles of the Suzuki-Miyaura coupling reaction of 4-bromonitrobenzene $\mathbf{1 a}$ and phenylboronic acid (Table S1 in the ESI $\dagger$ ). After each cycle, PAF70-Pd was easily recovered by centrifugation followed by washing and could be directly used in the next cycle for cycles $2-3$, in which the substrate dosages were the same as that in cycle 1. After 3 cycles, the recovered PAF70-Pd was dried in vacuo at $120^{\circ} \mathrm{C}$ for $18 \mathrm{~h}$. ICP analysis showed that the recovered PAF70-Pd had 22.7 wt $\%$ of Pd content, which had no obvious change compared with the fresh PAF70-Pd (23.0 wt\% of Pd content). These indicated that there is very low metal leaching during the reaction process. The results demonstrated that PAF70-Pd could undergo at least 3 cycles of the reaction without obvious loss of catalytic activity.

\subsection{Comparison with the previously reported porous organic material (POPs and COFs) immobilized Pd catalysts}

Data on the Pd contents and the TOF values of corresponding catalytic systems of PAF70-Pd, PAF1-Pd and previously reported porous organic material immobilized Pd catalysts are given in Fig. 3 and Table S2 in the ESI. $\dagger$ The TOF values were all calculated for the whole reaction process after complete conversion of the reactant under the respective optimum conditions. It is quite noticeable that PAF70-Pd and PAF1-Pd have the highest Pd contents, which might be due to the high effective surface areas of our materials. Moreover, PAF70-Pd showed far higher TOF values than other catalysts in Fig. 3. In addition, PAF70-Pd gave a rare example of a catalytic system with size selectivity in this field (Table S2 in the ESI $\dagger$ ). These excellent catalytic properties of PAF70-Pd could be attributed to the large enough remnant pore space after the introduction of the catalytic sites. That is, the remnant pore space could accommodate the reactants entering into the pores and the products exiting outside of the pores.

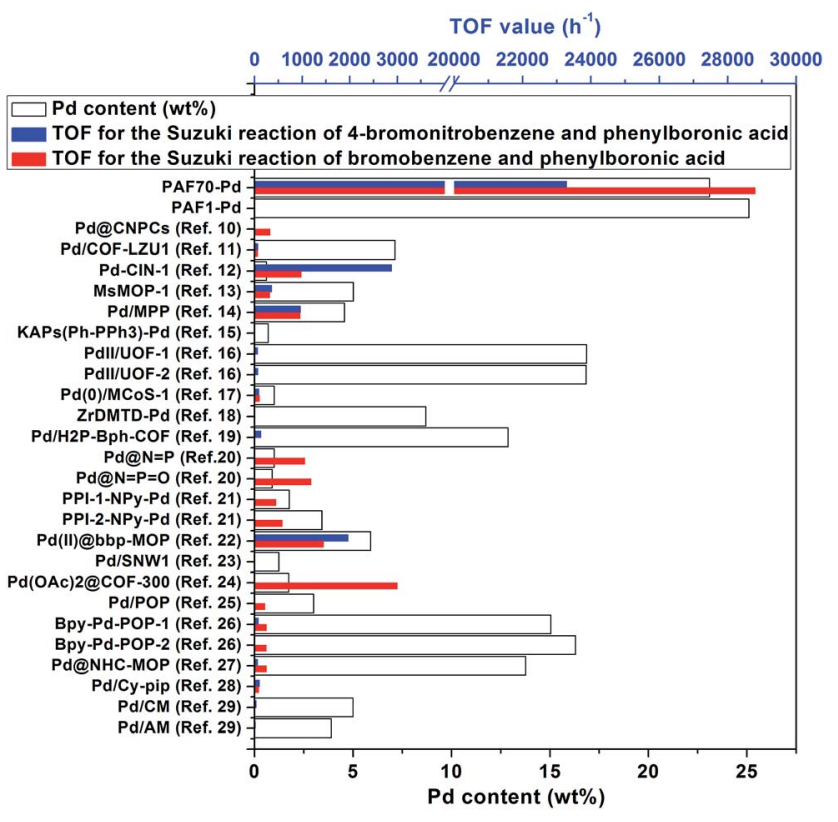

Fig. $3 \mathrm{Pd}$ contents and the catalytic performances in the SuzukiMiyaura coupling reaction of PAF70-Pd, PAF1-Pd and other reported porous organic material (POPs and COFs) immobilized Pd catalysts.

\section{Conclusions}

Based on the mesoporous PAF (PAF70-NH $\mathbf{N H}_{2}$ ), by a post-synthesis method, PAF70-N=CPy with an $N, N$-bidentate ligand was successfully obtained. After a second post-treatment with palladium acetate, the PAF material containing the Pd-based molecular catalyst, named PAF70-Pd, was prepared. Because the narrowly distributed mesopores $(3.8 \mathrm{~nm})$ in PAF70- $\mathbf{N H}_{2}$ endow sufficient pore space for immobilizing the Pd-based molecular catalyst with a relatively large size, the resulting immobilized catalyst PAF70-Pd has evenly distributed Pd species and high Pd content (23.0 wt\%). Furthermore, PAF70-Pd showed superb catalytic activity for catalyzing the Suzuki-Miyaura coupling reaction with good size selectivity and very easy recyclability. Compared with the reported porous organic material immobilized Pd catalysts, PAF70-Pd has the highest Pd content and exhibits a far higher TOF value when catalyzing the same Suzuki-Miyaura coupling reaction. By comparison with PAF1-Pd, it was clearly demonstrated that the mesopores in PAF70-NH $\mathbf{N H}_{2}$ are very important for the high activity of PAF70-Pd. After the introduction of Pd species with a relatively large size, PAF70-Pd still has large enough pore space to accommodate the catalyzed reaction. This could significantly enhance the utilization efficiency of the Pd catalyst in the material. Our strategy supplied a versatile method for the immobilization of different organometallic catalysts into the pores of PAFs, which will promote the development of PAF-based organometallic catalysts.

\section{Conflicts of interest}

There are no conflicts to declare. 


\section{Acknowledgements}

We are grateful for the financial support of the National Basic Research Program of China (973 Program, grant no. 2014CB931804) and the National Natural Science Foundation of China (NSFC Project, grant no. 21302061 and 21531003).

\section{Notes and references}

1 N. Kaur, Inorg. Chem. Commun., 2014, 49, 86-119.

2 N. Kaur, Catal. Rev., 2014, 57, 1-78.

3 I. Maluenda and O. Navarro, Molecules, 2015, 20, 7528-7557.

4 K. Okumura, H. Matsui, T. Tomiyama, T. Sanada, T. Honma, S. Hirayama and M. Niwa, ChemPhysChem, 2009, 10, 32653272 .

5 K. Okumura, T. Tomiyama, S. Okuda, H. Yoshida and M. Niwa, J. Catal., 2010, 273, 156-166.

6 P. Kaur, J. T. Hupp and S. T. Nguyen, ACS Catal., 2011, 1, 819-835.

7 Q. Sun, Z. Dai, X. Meng, L. Wang and F.-S. Xiao, ACS Catal., 2015, 5, 4556-4567.

8 H. Zhong, C. Liu, Y. Wang, R. Wang and M. Hong, Chem. Sci., 2016, 7, 2188-2194.

9 Á. Molnár and A. Papp, Coord. Chem. Rev., 2017, 349, 1-65. 10 P. Zhang, Z. Weng, J. Guo and C. Wang, Chem. Mater., 2011, 23, 5243-5249.

11 S. Y. Ding, J. Gao, Q. Wang, Y. Zhang, W. G. Song, C. Y. Su and W. Wang, J. Am. Chem. Soc., 2011, 133, 19816-19822.

12 M. K. Bhunia, S. K. Das, P. Pachfule, R. Banerjee and A. Bhaumik, Dalton Trans., 2012, 41, 1304-1311.

13 H. Li, B. Xu, X. Liu, S. A, C. He, H. Xia and Y. Mu, J. Mater. Chem. A, 2013, 1, 14108.

14 Q. Song, Y. Jia, B. Luo, H. He and L. Zhi, Small, 2013, 9, 24602465.

15 Z. Guan, B. Li, G. Hai, X. Yang, T. Li and B. Tan, RSC Adv., 2014, 4, 36437.

16 L. Li, Z. Chen, H. Zhong and R. Wang, Chem.-Eur. J., 2014, 20, 3050-3060.

17 A. S. Roy, J. Mondal, B. Banerjee, P. Mondal, A. Bhaumik and S. M. Islam, Appl. Catal., A, 2014, 469, 320-327.

18 B. Gui, K. K. Yee, Y. L. Wong, S. M. Yiu, M. Zeller, C. Wang and Z. Xu, Chem. Commun., 2015, 51, 6917-6920.

19 Y. Hou, X. Zhang, J. Sun, S. Lin, D. Qi, R. Hong, D. Li, X. Xiao and J. Jiang, Microporous Mesoporous Mater., 2015, 214, 108114.

20 X. Jiang, W. Zhao, W. Wang, F. Zhang, X. Zhuang, S. Han and X. Feng, Polym. Chem., 2015, 6, 6351-6357.

21 E. Rangel Rangel, E. M. Maya, F. Sánchez, J. G. de la Campa and M. Iglesias, Green Chem., 2015, 17, 466-473.

22 Q. Wen, T. Y. Zhou, Q. L. Zhao, J. Fu, Z. Ma and X. Zhao, Macromol. Rapid Commun., 2015, 36, 413-418.

23 M. Shunmughanathan, P. Puthiaraj and K. Pitchumani, ChemCatChem, 2015, 7, 666-673.

24 R. S. B. Gonçalves, A. B. V. de Oliveira, H. C. Sindra, B. S. Archanjo, M. E. Mendoza, L. S. A. Carneiro, C. D. Buarque and P. M. Esteves, ChemCatChem, 2016, 8, 743-750.
25 X. Ren, S. Kong, Q. Shu and M. Shu, Chin. J. Chem., 2016, 34, 373-380.

26 C.-A. Wang, Y.-F. Han, Y.-W. Li, K. Nie, X.-L. Cheng and J.-P. Zhang, RSC Adv., 2016, 6, 34866-34871.

27 C.-A. Wang, Y.-W. Li, X.-M. Hou, Y.-F. Han, K. Nie and J.-P. Zhang, ChemistrySelect, 2016, 1, 1371-1376.

28 Z.-L. Du, Q.-Q. Dang and X.-M. Zhang, Ind. Eng. Chem. Res., 2017, 56, 4275-4280.

29 Y. Monguchi, F. Wakayama, S. Ueda, R. Ito, H. Takada, H. Inoue, A. Nakamura, Y. Sawama and H. Sajiki, RSC Adv., 2017, 7, 1833-1840.

30 A. Thomas, Angew. Chem., Int. Ed., 2010, 49, 8328-8344.

31 Y. Zhang and S. N. Riduan, Chem. Soc. Rev., 2012, 41, 20832094.

32 A. Dhakshinamoorthy and H. Garcia, Chem. Soc. Rev., 2012, 41, 5262-5284.

33 Y. Xu, S. Jin, H. Xu, A. Nagai and D. Jiang, Chem. Soc. Rev., 2013, 42, 8012-8031.

34 X. Zou, H. Ren and G. Zhu, Chem. Commun., 2013, 49, 39253936.

35 Y. Zhang and J. Y. Ying, ACS Catal., 2015, 5, 2681-2691.

36 Q. Yang, Q. Xu and H. L. Jiang, Chem. Soc. Rev., 2017, 46, 4774-4808.

37 H. J. Jeon, J. H. Choi, Y. Lee, K. M. Choi, J. H. Park and J. K. Kang, Adv. Energy Mater., 2012, 2, 225-228.

38 K. Konstas, J. W. Taylor, A. W. Thornton, C. M. Doherty, W. X. Lim, T. J. Bastow, D. F. Kennedy, C. D. Wood, B. J. Cox, J. M. Hill, A. J. Hill and M. R. Hill, Angew. Chem., Int. Ed., 2012, 51, 6639-6642.

39 W. Lu, J. P. Sculley, D. Yuan, R. Krishna, Z. Wei and H.-C. Zhou, Angew. Chem., Int. Ed., 2012, 51, 7480-7484.

40 B. Li, Y. Zhang, R. Krishna, K. Yao, Y. Han, Z. Wu, D. Ma, Z. Shi, T. Pham, B. Space, J. Liu, P. K. Thallapally, J. Liu, M. Chrzanowski and S. Ma, J. Am. Chem. Soc., 2014, 136, 8654-8660.

41 L. N. Li, H. Ren, Y. Yuan, G. L. Yu and G. S. Zhu, J. Mater. Chem. A, 2014, 2, 11091-11098.

42 C. H. Lau, K. Konstas, C. M. Doherty, S. Kanehashi, B. Ozcelik, S. E. Kentish, A. J. Hill and M. R. Hill, Chem. Mater., 2015, 27, 4756-4762.

43 Z. Yan, Y. Yuan, Y. Tian, D. Zhang and G. Zhu, Angew. Chem., Int. Ed., 2015, 54, 12733-12737.

44 S. Demir, N. K. Brune, J. F. Van Humbeck, J. A. Mason, T. V. Plakhova, S. Wang, G. Tian, S. G. Minasian, T. Tyliszczak, T. Yaita, T. Kobayashi, S. N. Kalmykov, H. Shiwaku, D. K. Shuh and J. R. Long, ACS Cent. Sci., 2016, 2, 253-265.

45 B. Li, Y. Zhang, D. Ma, Z. Xing, T. Ma, Z. Shi, X. Ji and S. Ma, Chem. Sci., 2016, 7, 2138-2144.

46 H. Zhao, Z. Jin, H. Su, J. Zhang, X. Yao, H. Zhao and G. Zhu, Chem. Commun., 2013, 49, 2780-2782.

47 C. H. Lau, K. Konstas, A. W. Thornton, A. C. Liu, S. Mudie, D. F. Kennedy, S. C. Howard, A. J. Hill and M. R. Hill, Angew. Chem., Int. Ed., 2015, 54, 2669-2673.

48 C. A. Wang, Z. K. Zhang, T. Yue, Y. L. Sun, L. Wang, W. D. Wang, Y. Zhang, C. Liu and W. Wang, Chem.-Eur. J., 2012, 18, 6718-6723. 
49 E. Merino, E. Verde-Sesto, E. M. Maya, M. Iglesias, F. Sánchez and A. Corma, Chem. Mater., 2013, 25, 981-988.

50 E. Merino, E. Verde-Sesto, E. M. Maya, A. Corma, M. Iglesias and F. Sánchez, Appl. Catal., A, 2014, 469, 206-212.

51 Y. Zhang, B. Li and S. Ma, Chem. Commun., 2014, 50, 85078510.

52 Y. Wang, L. Wang, C. Liu and R. Wang, ChemCatChem, 2015, 7, 1559-1565.
53 E. Rangel-Rangel, E. Verde-Sesto, A. M. Rasero-Almansa, M. Iglesias and F. Sánchez, Catal. Sci. Technol., 2016, 6, 6037-6045.

54 E. Verde-Sesto, E. Merino, E. Rangel-Rangel, A. Corma, M. Iglesias and F. Sánchez, ACS Sustainable Chem. Eng., 2016, 4, 1078-1084.

55 J. S. Sun, L. P. Jing, Y. Tian, F. Sun, P. Chen and G. Zhu, Chem. Commun., 2018, 54, 1603-1606.

56 M. Murakami and T. Matsuda, Chem. Commun., 2011, 47, 1100-1105. 